\title{
Experimental determination of some physical properties of gasoline, ethanol and ETBE ternary blends
}

\author{
Luis Miguel Rodríguez Antón , Fernando Gutiérrez Martín, Carmen Martinez Arevalo
}

\begin{abstract}
A B S T R A C T
The addition of oxygenated renewable fuels, such as ethanol or ethyl tert-butyl ether (ETBE) to standard gasoline may be necessary to comply with some environmental directives but could also prevent compliance with some fuel regulations and could also seriously change engine performance. From this point of view, the Reid Vapour Pressure (RVP), the distillation curve, the oxygen content and the density belong to the group of the most relevant parameters. This study evaluates the influence of the simultaneous addition of ethanol and ETBE on some physical properties of engine gasoline. The main conclusion is that the simultaneous addition of ETBE and ethanol changes the RVP, the distillation curve and the density in a way that can affect engine operation and the mandatory EN 228 and ASTM D4814 standards. Some opposite properties of both oxygenates could help to increase the renewable energy content without preventing compliance with these regulations.
\end{abstract}

\section{Introduction}

From the year 2011 to 2030 , global energy consumption is projected to grow by $36 \%$ to 16.6 billion toe and the energy consumption in transport by $27 \%$ to 2.7 billion toe [1]. To meet this demand, a diverse energy mix is needed. This is the point where biofuels could help; in the next two decades, biofuels are expected to provide some $13 \%$ (by energy content) of the growth in fuels for road transport [1]. Additionally, due to the fast-growing concern about global warming, which can be attributed primarily to the elevated $\mathrm{CO}_{2}$ levels in the atmosphere, the use of renewable energy is mandatory.

That is the reason why many countries have developed fuel regulations in order to reduce, first, their carbon dioxide emissions into the atmosphere and, second, their foreign energy dependence. In this way, the Directive $2009 / 28 / \mathrm{EC}$ [2] requires $10 \%$ of renewable content for transport fuels before 2020 and higher percentages are foreseeable in the coming years. In parallel with these Directives, fuel regulations are being developed to allow the addition of some renewable components [3].

Alcohols have been used as fuels for engines since the 19th century. Among the various alcohols, ethanol ( $100 \%$ renewable) is known as the most suitable renewable, bio-based and eco-friendly fuel for spark-ignition engines [4]. Nevertheless, isobutanol also has good future perspectives $[5,6]$. On the other hand, ETBE $(47 \%$ renewable) is one of the most common ethers used as a fuel additive [7]. Nowadays, most advanced countries add either ethanol and/or ETBE to their gasoline pools [7-16].

When ethanol and/or ETBE are added to gasoline, some fuel properties change and the gaseous emissions, the mechanical performance and the reliability of the engine could be affected either positively or negatively. To guarantee compliance with new mandatory renewable fuel contents, but also to maintain good 
engine performance, changes in fuel specifications must be made step-by-step.

It is well known that the RVP, the distillation curve and the oxygen content, as well as the octane number, belong to the group of fuel properties that affect engine operation and gaseous emissions more significantly. Any changes to these properties could affect engine behaviour negatively, if no precautions are taken. That is the reason why many researchers have looked into changes in these properties when ethanol and/or ETBE are added to gasoline, by empirical or theoretical determination.

Muzíkovâ [8] has studied the influence of ethanol up to $10 \% \mathrm{v} / \mathrm{v}$, ETBE up to $10 \% \mathrm{v} / \mathrm{v}$, as well as hydrocarbon composition on the volatility and distillation characteristics of the gasoline blends. Karonis [9] has examined the impact of simultaneous addition of ETBE up to $6 \% \mathrm{v} / \mathrm{v}$ and ethanol up to $6 \% \mathrm{v} / \mathrm{v}$ on the main properties of motor gasoline (RVP, E70, E100, E150, RON, MON, and sensitivity). Andersen $[10,11]$ has determined the RVP and the distillation curves for various alcohols-gasoline binary blends containing up to $85 \% \mathrm{v} / \mathrm{v}$ of ethanol. This author has reported a simple method to prepare two-alcohols-gasoline blends with a RVP that is indistinguishable from that of the base gasoline and has also demonstrated that those blends have distillation curves closer to that of the base gasoline than single-alcohol blends. Christensen [12] has studied the chemical and physical properties (RVP, vapour lock protection, distillation, density, octane rating, viscosity and potential for extraction into water) for various alcohol-gasoline blends of up to $3.7 \% \mathrm{w} / \mathrm{w}$ of oxygen and has compared them with the requirements of the ASTM D4814 specification to determine their utility as gasoline extenders. Petre $[13,14]$ has used two classical gasolines with different composition and properties, with proportions ranging from $2 \%$ to $10 \% \mathrm{v} / \mathrm{v}$ of ethanol and other alcohols, plus addition of ETBE in proportions ranging from $4 \%$ to $15 \% \mathrm{v} / \mathrm{v}$ and other ethers; she has presented experimental results regarding the effect of the blend with alcohol or ether on the RVP and the distillation curve. Pumphrey [17] has reported a simple method to successfully predict vapour pressures of gasoline-alcohol mixtures and validate them with blends of gasoline with different oxygenates $(0-100 \% \mathrm{v} / \mathrm{v})$. French [16] has shown the effect of the addition of ethanol to gasoline on the RVP, the distillation curves $(0-10 \% \mathrm{v} / \mathrm{v})$, the Vapour/Liquid ratio (V/L), the Vapour Lock Index (VLI), the Driveability Index (DI), the phase separation and the material compatibility. Menezes [7] has analysed the effect of the addition of an azeotropic ETBE/ethanol mixture in two gasolines $(0-17 \% \mathrm{v} / \mathrm{v})$ on the RVP, the distillation curve, the density and the octane number. Zemroch [18] has determined what happens to the volatility of the ethanol/gasoline blend up to $25 \% \mathrm{v} / \mathrm{v}$, specifically its E70, E100 and RVP, when ethanol is splash blended into a wide range of base gasolines.

Many other researchers have studied the influence of the addition of oxygenates to gasoline on engine performance, mainly focusing on the gaseous emissions [15,19-23], torque, power, fuel economy [19-21,24], material compatibility [25], driveability or engine operability and reliability.

For all the above reasons, the interest in this matter nowadays seems evident. The high octane number of ethanol or ETBE means they are suitable as replacements for alkyl-lead compounds as gasoline octane improvers, owing to environmental and public health reasons [16]. The main objective of this paper is to offer reliable experimental data of the distillation curves, the RVP and the density of ternary blends of gasoline, ethanol and ETBE. These properties, as well as the chemical composition of the base gasoline allow the authors to determine most of the regulated parameters in the Directive 2009/30/CE [3] and check their compliance with the mandatory specifications (EN 228, ASTM D4814). The knowledge of these data could help researchers to foresee the engine performance when these ternary blends are used and to determine their optimum composition. Throughout this paper, the influence of each parameter over engine performance will be presented.

\section{Materials and methods}

To carry out this research some fuels and experimental facilities that comply with European standards were used.

\subsection{Materials}

The base gasoline (Table 1), Ethanol and ETBE have already been used in a previous work where their specifications were stated [26].

To prepare the gasoline-ethanol-ETBE blends, some ' $A$ ' class pipettes, beakers and volumetric flasks with the maximum precision (DIN 12600) were used. The abbreviated blend names were built with two letters ( $\mathrm{E}$ and $\mathrm{T}$ ) and two numbers between 0 and 100 . The letter ' $E$ ' precedes the volume percentage of added ethanol and the letter ' $\mathrm{T}$ ' precedes the volume percentage of added ETBE (ETBE*). For example: 'E20T15' means 20\% v/v of ethanol, $15 \% \mathrm{v} / \mathrm{v}$ of ETBE ${ }^{*}$ and $65 \% \mathrm{v} / \mathrm{v}$ of base gasoline. In this study, base gasoline contained $0.1 \% \mathrm{v} / \mathrm{v}$ of ethanol, which is negligible, and $6 \% \mathrm{v} / \mathrm{v}$ of ETBE. It would have been better to use a base gasoline without ETBE and ethanol but it is not currently possible to find it on the market.

The tested blends for densities, RVP and distillation curves were all possible combinations of base gasoline, ethanol $(0,2,4,6,8,10$, $15,20,30,45,60$ and $85 \% \mathrm{v} / \mathrm{v})$ and $\operatorname{ETBE}(5,10,15,20,25$ and $30 \%$ $\mathrm{v} / \mathrm{v}$ ). Note that some combinations as for example T30E85 or T25E85 are not possible. In the case of distillation curves, the ethanol-ETBE binary blends were not tested.

\subsection{Methods: experimental facilities and test management}

The tests were carried out in the 'Fuels and Petrochemical Laboratory' of the 'Gómez Pardo Foundation'. This official Spanish laboratory is accredited by ENAC (National Entity of Accreditation) by the UNE-EN ISO/IEC 17025 standard. The density, RVP and distillation curves were measured following the UNE-EN ISO 12185, UNE-EN 13016-1 and UNE-EN ISO 3405 standards, respectively. All the tests were carried out according to corresponding equipment instructions. Their main technical specifications were described in the aforementioned work [26].

\section{Results and discussion}

In this section the main results obtained from the tests (RVP, distillation curve, density and composition) are presented and discussed.

\subsection{Density}

Solutions created by mixing ethanol and ETBE with gasoline are not ideal because the intermolecular forces and molecular packing of the same substance (ethanol-ethanol, gasoline-gasoline or ETBE-ETBE) are different from those of different substances (gasoline-ethanol-ETBE). Thus, the volume of the mixture is different from the sum of its components' volumes and this difference is called 'excess volume'. It seems to be clear that when ethanol is blended with gasoline, the intermolecular forces are reduced because ethanol is a polar substance and gasoline is not, though nothing can be guaranteed concerning the molecular packing. The case of ETBE, less polar than ethanol, is similar. If the excess volume is positive, the solution density would be less than ideal, 
Table 1

Heating values, properties, requirements and test methods for base gasoline with maximum oxygen content $2.7 \%$ (EN 228)

\begin{tabular}{|c|c|c|c|c|c|}
\hline Property & Units & Experimental value & Minimum limit & Maximum limit & Test method \\
\hline Density at $15^{\circ} \mathrm{C}$ & $\mathrm{kg} / \mathrm{m}^{3}$ & 737.6 & 720 & 775 & EN ISO 3675 \\
\hline Vapour Pressure (Summer - Class A -) & $\mathrm{kPa}$ & 60.5 & 45 & $60\left(^{a}\right)$ & EN ISO 13016-1 \\
\hline \multicolumn{6}{|l|}{ Distillation } \\
\hline Evaporated at $70^{\circ} \mathrm{C}(\mathrm{E} 70)$ & $\% v / v$ & 30.5 & 20 & 48 & EN ISO 3405 \\
\hline Evaporated at $100^{\circ} \mathrm{C}(\mathrm{E} 100)$ & $\% v / v$ & 60.0 & 46 & 71 & \\
\hline Evaporated at $150^{\circ} \mathrm{C}(\mathrm{E} 150)$ & $\% v / v$ & 90.7 & 75 & - & \\
\hline Final Boiling Point (FBP) & ${ }^{\circ} \mathrm{C}$ & 195.2 & - & 210 & \\
\hline Distillation Residue (DR) & $\% v / v$ & 0.6 & - & 2 & \\
\hline \multicolumn{6}{|l|}{ Hydrocarbon type content } \\
\hline Olefins & $\% \mathrm{v} / \mathrm{v}$ & 13.09 & - & 18 & EN14517 EN15553 \\
\hline Aromatics & $\% v / v$ & 27.05 & - & 35 & \\
\hline Benzene & $\% v / v$ & 0.86 & - & 1 & UNE EN238 \\
\hline \multicolumn{6}{|l|}{ Oxygen and Oxygenates content } \\
\hline Oxygen content & $\% w / w$ & 0.98 & - & 2.7 & UNE EN 13132 \\
\hline Ethanol & $\% v / v$ & 0.1 & - & 5 & EN 13132 \\
\hline Ethers ( 5 or more $C$ atoms) & & 6 & - & - & \\
\hline \multicolumn{6}{|l|}{ Sulphur and Copper content } \\
\hline Sulphur content & $\mathrm{mg} / \mathrm{kg}$ & 7.0 & - & 10 & EN ISO 20846 \\
\hline Copper strip corrosion $\left(3 \mathrm{~h}\right.$ at $\left.50^{\circ} \mathrm{C}\right)$ & Rating & Class 1 & - & Class 1 & EN ISO 2160 \\
\hline \multicolumn{6}{|l|}{ Heating values } \\
\hline Higher & $\mathrm{kJ} / \mathrm{kg}$ & 43238 & - & - & \\
\hline Lower & $\mathrm{kJ} / \mathrm{kg}$ & 41048 & - & - & \\
\hline
\end{tabular}

${ }^{a}$ Extra vapour pressure is permitted depending on $\mathrm{EtOH}$ content.

and vice versa. To evaluate if the excess volume is relevant when gasoline, ethanol and ETBE are mixed, the densities of all the ternary mixtures were measured. The difference between the experimental and the ideal density (weighted mean by volumetric composition of gasoline, ethanol and ETBE densities) was slightly different but always less than $0.5 \%$. Thus, it could be assumed that the density of a mixture is the weighted mean by volumetric composition of the components' densities $\left(737.6 \mathrm{~kg} / \mathrm{m}^{3}\right.$ for the base gasoline, $794.0 \mathrm{~kg} / \mathrm{m}^{3}$ for ethanol and $744.2 \mathrm{~kg} / \mathrm{m}^{3}$ for ETBE). This approximation provided a coefficient of determination of $99.93 \%$.

It is important to remark that the higher density of both oxygenated additives, as compared with base gasoline $0.89 \%$ for ETBE and $7.65 \%$ for ethanol), slightly compensates for their lower heat value (13.0\% for ETBE and $35.4 \%$ for ethanol), due to their oxygen content. The higher density of both oxygenates, especially ethanol, caused ethanol concentrations which were greater than $65 \% \mathrm{v} / \mathrm{v}$ to surpass the density upper limit fixed by the EN 228 standard.

\subsection{Reid Vapour Pressure (RVP)}

RVP has a great influence on evaporative hydrocarbon emissions from the fuel tank of vehicles. This is the reason why all regulations around the world limit this parameter depending on climatic conditions.

The RVP of ETBE-gasoline mixtures was almost ideal, that is, it was the weighted mean by molar fraction composition of ETBE and gasoline RVP. It should be observed that molar and volume fractions have approximately the same values in the case of ETBE-gasoline blends [26].

The behaviour of ethanol-gasoline mixtures is significantly different. Despite the fact that the RVP of ethanol is much lower than that of gasoline (because of the stronger intermolecular hydrogenbonding interactions between ethanol molecules), some mixtures of these fuels increase the RVP of the blend. This positive deviation from the ideal mixture behaviour (Raoult's Law) occurs because the intermolecular interactions between ethanol and hydrocarbon molecules are weaker than they are in the two pure liquids [27] and also due to the formation of azeotropic mixtures between ethanol and lighter fractions of gasoline (n-butane, n-pentane, etc.) that reduce the boiling point temperature and increase the vapour pressure of the mixture [18]. These facts have been confirmed by analysing the vapour of different ethanol-ETBE-gasoline mixtures by gas chromatography [26].

When the two oxygenates were added simultaneously, the behaviour of ternary blends was not ideal. The deviation from the ideal mixture was positive for the reasons mentioned above. Fig. 1 shows a map of RVP as a function of ethanol and ETBE* content. The maximum RVP took place between $5 \%$ and $10 \% \mathrm{v} / \mathrm{v}$ of ethanol, while any addition of ETBE reduced the value of RVP. The increase of RVP due to the addition of $5-10 \% \mathrm{v} / \mathrm{v}$ of ethanol ( $\sim 6-8 \mathrm{kPa}$ [27]) can be compensated by adding about $15 \% \mathrm{v} / \mathrm{v}$ of ETBE $^{*}$ (E8T15). Higher quantities of added ethanol required lower quantities of ETBE*. This corrector effect of ETBE could help to comply with the EN 228 or ASTM D4814 standards. In any case, Fig. 1 shows that higher amounts of ethanol and ETBE could surpass the limits marked by the standards for the total oxygen content.

Finally, the measured data have been adjusted to a quadratic surface giving a coefficient of determination of $98.98 \%$ and a maximum deviation of $\pm 3 \mathrm{kPa}$.

\subsection{Distillation curve}

The distillation curve profile affects engine behaviour significantly. Prakash [28] describes it in a detailed way. The evaporative losses from the tank, the hot or cool engine starting and the vapour lock depend on lighter fractions ( $0 \%$ to $40 \%$ of distilled volume). The ice formation in intake air system, the engine warm-up, the vehicle acceleration and the short-trip fuel economy depend on medium fractions ( $\sim 40 \%$ to $\sim 80 \%$ of distilled volume). Finally, the long-trip economy, the oil dilution, the volatile organic compounds (VOC) and the formation of combustion deposits depend on heavy fractions ( $\sim 80 \%$ to $100 \%$ of distilled volume).

The strong influence that the distillation curves have on the engine performance means that fuel regulations must have many parameters to control these curves. European regulations (EN 228 ) control the volume evaporated at 70,100 and $150^{\circ} \mathrm{C}$ (E70/ E100/E150, abbreviations which should not be confused with those 
VAPOUR PRESSURE [kPa]

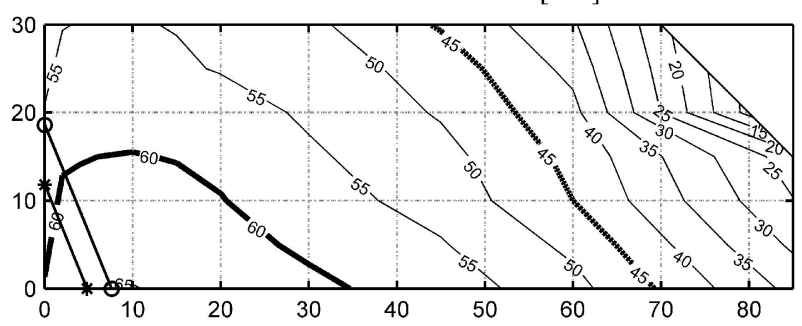

VAPOUR LOCK [INDEX]

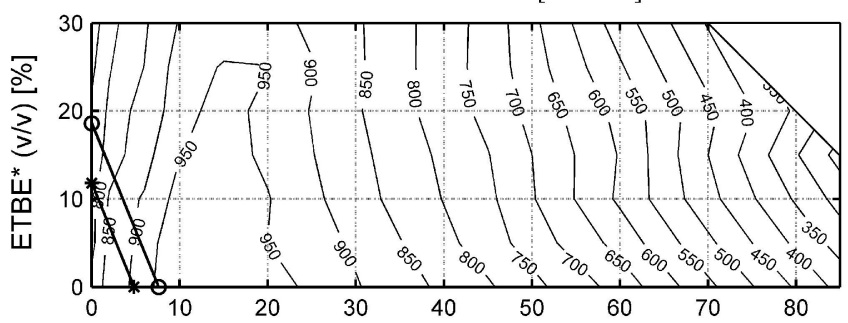

DRIVEABILITY [INDEX]

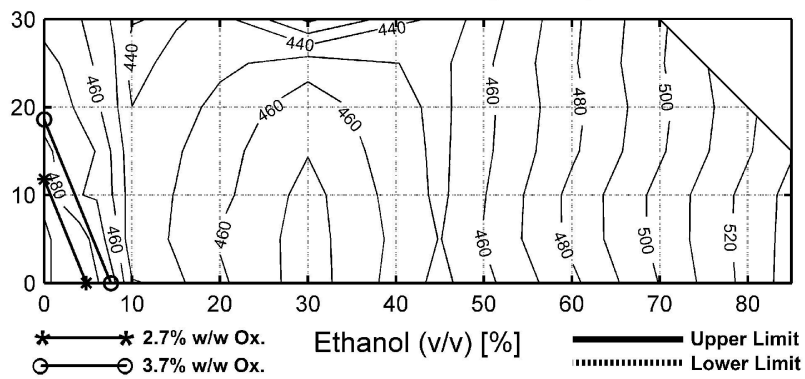

Fig. 1. RVP, DI and VLI vs. composition of ethanol, ETBE* and gasoline ternary blends.

referring to the mixtures of ethanol with gasoline, mentioned above), the Vapour Lock Index $(V L I=10 \times R V P+7 \times E 70)$, the Distillation End Point (DEP) and the Distillation Residue (DR). American regulations (ASTM D4814) control the temperatures at which $10 \%, 50 \%$ and $90 \%$ of fuel volume are evaporated (T10/T50/ T90, which should not be confused with those referring to the mixtures of ETBE and gasoline), the Driveability Index $(D I=1.5 \times$ $T 10+3.0 \times T 50+1.0 \times T 90+1.33 \times \%$ Ethanol $v / v)$, the DEP and the DR.

The study of volatility curves for all the blends prepared in this work was a hard task due to the high number of measurements. To better understand this hard task, it is advisable to see the volatility curves of the same base gasoline blended only with ETBE (Fig. 2 [26]) or ethanol (Figure 4 [26]). It was more practical to analyse the changes in the parameters controlled by the standards (E70, E100, E150, VLI, FP and DR for EN 228 or T10, T50, T90, DI, FP and DR for ASTM D4814). The actual limits for the geographical zone and season when the base gasoline was purchased are summarized in Table 2. In the following paragraphs, the influence of the addition of ethanol and ETBE on the above parameters is discussed.

The fixed limits for DR and DEP are aimed at controlling the formation of engine deposits, the VOC's emissions and the fuel dilution in the oil. The addition of both oxygenates reduced both parameters and thus, the obtained values are lower than the values of the base gasoline and the upper limits.

The VLI tries to control the problems associated with vapour formation in the fuel lines. In modern engines, this parameter is not usually a problem because these engines operate with fuel
E70 [\%]

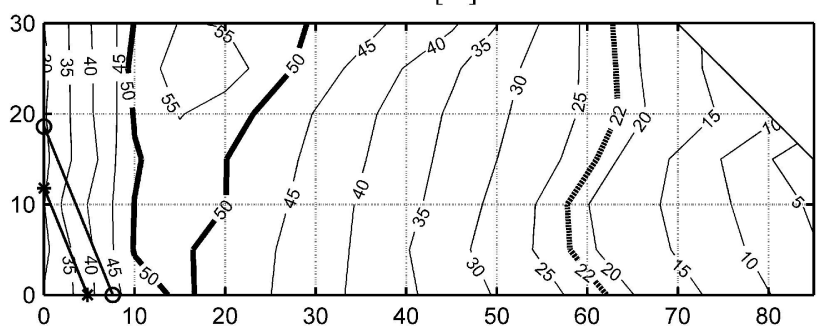

E100 [\%]

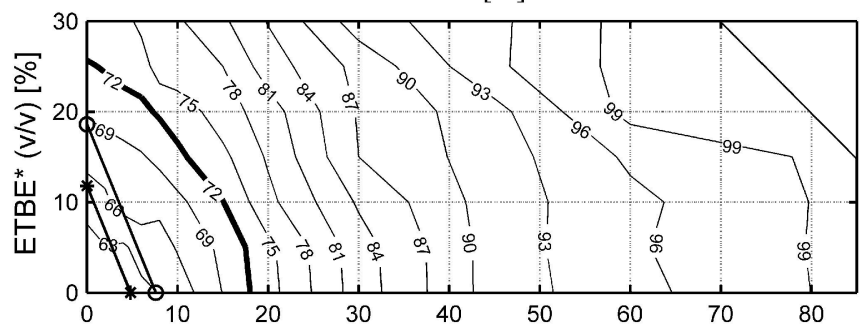

E150 [\%]



Fig. 2. Volatility parameters of EN 228 (E70, E100, E150) for ethanol, ETBE* and gasoline ternary blends.

Table 2

RVP and requirements (EN 228 and ASTM D4814) for the gasoline distillation curve (volatility class A).

\begin{tabular}{lllll}
\hline Standard & Parameter & Units & Min & Max \\
\hline $\begin{array}{l}\text { EN } 228 \text { (Distillation class A and Oxygen } \\
\text { content 3.7\% w/w) }\end{array}$ & RVP & $\mathrm{kPa}$ & 45 & 60 \\
& & & & \\
& E70 & $\% \mathrm{v} / \mathrm{v}$ & 22 & 50 \\
& E100 & $\% \mathrm{v} / \mathrm{v}$ & 46 & 72 \\
& E150 & $\% \mathrm{v} / \mathrm{v}$ & 75 & \\
& End Point & ${ }^{\circ} \mathrm{C}$ & 210 \\
& Residue & $\% \mathrm{v} / \mathrm{v}$ & 2 \\
ASTM 4814 (Distillation class A) & VLI & Index & - & - \\
& RVP & $\mathrm{kPa}$ & 62 \\
& T10 & ${ }^{\circ} \mathrm{C}$ & 70 \\
& T50 & ${ }^{\circ} \mathrm{C}$ & 66 & 121 \\
& T90 & ${ }^{\circ} \mathrm{C}$ & 190 \\
& End Point & ${ }^{\circ} \mathrm{C}$ & 225 \\
& Residue & $\% \mathrm{v} / \mathrm{v}$ & 2 \\
& DI & ${ }^{\circ} \mathrm{C}$ & 597 \\
\hline
\end{tabular}

pumps immersed in the tank and with pressurized fuel lines. The VLI presented a maximum value when ethanol concentration was around $15 \% \mathrm{v} / \mathrm{v}$, as shows Fig. 1 . The addition of ETBE slightly reduced the VLI. The maximum value (997.3), reached for E15T0, was much lower than the upper limit of the EN 228 standard (there is no limit for the summer gasoline tested, Class A, and the limit is 1068 for the winter gasoline, Class C).

Although each range of a distillation profile is important, the gasoline represented by an entire profile is what the engine must distribute, vaporize, and burn. To predict a fuel's cold-start and 
warm-up driveability, a driveability index (DI) has been developed (ASTM D4814) using T10, T50, T90 and ethanol content [29]. Lower values of DI generally result in better cold-start and warm-up performance. The addition of ethanol and ETBE slightly reduced the DI value with respect to the base gasoline, except for ethanol concentrations higher than $65 \% \mathrm{v} / \mathrm{v}$. In any case, this value was always lower than the upper limit (597) fixed by the ASTM D4814, therefore no engine problems were expected with this parameter (Fig. 1).

To control the gasoline composition (light, medium and heavy fractions respectively) the parameters used are E70, E100 and E150 in EN 228 standard and T10, T50 and T90 in ASTM D4814 standard. The addition of ethanol and ETBE substantially modified these parameters, especially those referring to light and medium fractions.

The addition of ETBE to gasoline involves lower volatility for lighter fractions and higher volatility for medium and heavy fractions.

The case of ethanol is very different as it forms azeotropic mixtures with many hydrocarbon compounds of gasoline, especially the lightest, having a lower boiling point than ethanol and the corresponding hydrocarbons $[30,31]$. The addition of ethanol to gasoline involves higher or lower volatility for lighter fractions depending on ethanol concentration. Medium and heavy fractions of the blends are always more volatile than those of base gasoline.

The parameter E70 was practically unaffected by the addition of ETBE in the range from $0 \%$ to $10 \%$ of ethanol. For higher concentrations of ethanol, the addition of ETBE slightly increased the value of E70. The addition of ethanol to the gasoline affected E70 in an important way, surpassing the EN 228 standard upper limit (50\%) for blends around E10-E20, and giving lower values than the lower limit (22\%) for blends with more than approximately $60 \% \mathrm{v} / \mathrm{v}$ of ethanol (Fig. 2).

The E100 parameter increased similarly for both oxygenates. The permitted upper limit (72\%) was reached with relatively low concentrations of oxygenates (E18T0, E10T17, E0T26) and higher concentrations substantially surpassed this upper limit (Fig. 2).

The E150 parameter increased similarly with the addition of both oxygenates too, but it does not pose any problem with the standard, being far away from the lower limit (75\%) fixed in EN 228 (Fig. 2).

The T10 parameter increased also with the addition of both oxygenated compounds, although the addition of ethanol at low concentrations showed a minimum around E10. The upper limit $\left(70^{\circ} \mathrm{C}\right)$ is reached with high oxygenate content (E80T0, E75T10) and thus the use of medium or low concentrations does not represent a problem (Fig. 3).

The T50 is the parameter affected in the most significant way. Ethanol addition up to $10 \% \mathrm{v} / \mathrm{v}$ drastically reduced this value, higher concentrations, however, slightly increased it. On the other hand ETBE addition slightly reduced this parameter. The combined action of both oxygenates does not in any case surpass the lower limit $\left(66^{\circ} \mathrm{C}\right)$ fixed in the ASTM D4814 standard (Fig. 3).

The T90 parameter is reduced similarly by the action of both additives, and thus, this is not a problem with the standard, being far away from the fixed upper limit $\left(190^{\circ} \mathrm{C}\right)$ (Fig. 3). In every one of the former parameters, the limits were reached with blends having oxygen contents higher than the upper values fixed by both standards, depending on the gasoline type (see the $2.7 \%$ and $3.7 \% \mathrm{w} /$ $\mathrm{w}$ oxygen lines in the figures).

\subsection{Composition-related parameters}

The chemical analysis of the base gasoline provided the elementary following formula: $\mathrm{C}_{7.18} \mathrm{H}_{12.72} \mathrm{O}_{0.06}$ and confirmed the compliance with the EN 228 standard regarding the limits of sulphur,
$\mathrm{T} 10\left[{ }^{\circ} \mathrm{C}\right]$
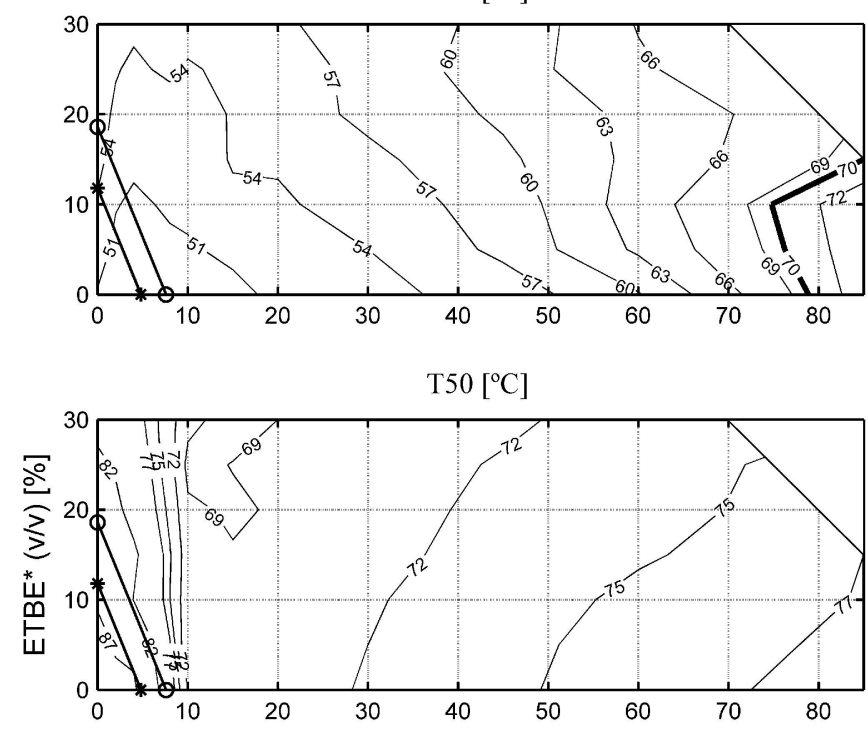

$\mathrm{T} 90\left[{ }^{\circ} \mathrm{C}\right]$



Fig. 3. Volatility parameters of ASTM D4814 (T10, T50, T90) for ethanol, ETBE* and gasoline ternary blends.

oxygen and benzene content (Table 1). Because of the absence of sulphur and benzene in ethanol or ETBE samples, all blends of these oxygenates with gasoline complied with the EN 228 and ASTM D4814 standards regarding the limits for these substances.

The oxygen content in ETBE and ethanol is $15.66 \%$ and $34.73 \%$ $\mathrm{w} / \mathrm{w}$ respectively. Consequently, the addition of ETBE and ethanol increases the oxygen content of the blend and could surpass the EN 228 and ASTM D4814 oxygen content limit. This parameter has a special relevance because it strongly affects the combustion stoichiometry, impeding the correct functioning and greatly changing the gaseous emissions and fuel economy of some engines.

Figs. $1-3$ show the $2.7 \%$ and $3.7 \% \mathrm{w} / \mathrm{w}$ oxygen content lines. It is necessary to keep in mind that the base gasoline had $6 \% \mathrm{v} / \mathrm{v}$ of ETBE, which is equivalent to around $2.7 \% \mathrm{v} / \mathrm{v}$ of ethanol. From these figures it is possible to calculate that $8 \%$ of total ethanol or $17 \%$ of total ETBE surpasses the real EN 228 limit $(2.7 \% \mathrm{w} / \mathrm{w})$. The recent increase of this limit to $3.7 \% \mathrm{w} / \mathrm{w}$ extends the use of biofuels up to $10 \% \mathrm{v} / \mathrm{v}$ of total ethanol or $23 \% \mathrm{v} / \mathrm{v}$ of total ETBE.

\section{Conclusions}

The main conclusion of this work is that physical properties of ethanol, ETBE and gasoline ternary blends change some important parameters controlled by the regulations as they affect engine performance and gaseous emissions.

The volume excess of these ternary blends was so small (less than $0.5 \%$ ) that it could be considered insignificant. Therefore, 
the blend density could be calculated as the weighted mean of the volumetric blend composition. The high ethanol density implied that high concentrations of this additive $(>65 \% \mathrm{v} / \mathrm{v})$ surpassed the upper limits of the EN 228 standard.

The addition of ETBE to gasoline reduced the RVP in a way that is directly proportional to the ETBE content, but the addition of ethanol increased the RVP of the blend only up to $35 \% \mathrm{v} / \mathrm{v}$. Thus, the addition of ETBE helps to compensate for the RVP increase produced by the ethanol at low concentrations, although the combined addition of both oxygenates could surpass the upper oxygen content limit. Additionally, any oxygenates content higher than E70T0-E45T30 surpass the lower limit for the EN 228 standard ( $45 \mathrm{kPa}$ ).

Regarding the distillation curves, the parameters most affected by the addition of oxygenates were those characterizing the light (E70) and medium (E100) fractions in the EN 228 standard. The lighter fractions did not fulfil the standards for ethanol content around $10-20 \% \mathrm{v} / \mathrm{v}$ or higher than $60-75 \% \mathrm{v} / \mathrm{v}$. The medium fractions (E100) surpassed the upper limit at relatively low oxygenate content (E18T0, E10T17, E0T26). The heavy fractions fulfilled both standards for any oxygenate content.

The total oxygen content $(2.7 \%$ or $3.7 \% \mathrm{w} / \mathrm{w})$ was the most restrictive parameter in the EN 228 and ASTM D4814 standards, followed by RVP, E100 and E70.

\section{Acknowledgments}

This work was sponsored by the 'Fuels and Petrochemical Laboratory' of the 'Gómez Pardo' Foundation. The authors gratefully thank the undergraduate students Julia Gómez and Óscar del Saz of the Universidad Politécnica of Madrid for carrying out the laboratory tests. The authors also wish to thank REPSOL and Acciona Energy for providing the fuel additives tested. 